Dixis Figueroa Pedraza ${ }^{1,2}$

1 Universidade Estadual da Paraíba, Programa de PósGraduação em Saúde Pública. Campina Grande, PB, Brasil.

${ }^{2}$ Rede Nordeste de Formação em Saúde de Família, Mestrado Profissional em Saúde da Família. Natal, RN, Brasil.

Correspondência Dixis Figueroa Pedraza dixisfigueroa@gmail.com

\section{Duração do aleitamento materno e sua associação com características maternas e orientações sobre incentivo à amamentação recebidas no pré-natal em unidades básicas de Saúde da Família de um município do Nordeste brasileiro}

Duration of breastfeeding and its association with maternal characteristics and counseling for the promotion of breastfeeding received in primary family health care units in a town in the Brazilian Northeast

\footnotetext{
Resumo

Objetivo: Estimar o tempo de aleitamento materno entre crianças atendidas na rede pública de saúde e verificar diferenças segundo características maternas e da atenção pré-natal (número de consultas e orientações profissionais). Métodos: Pesquisa transversal realizada em 16 unidades básicas de Saúde da Família com 204 crianças nascidas em 2009, em um município do Nordeste brasileiro. A coleta de dados ocorreu de forma retrospectiva em 2011, por aplicação de questionário às mães das crianças. Utilizou-se a análise de sobrevida para estimar a duração mediana de aleitamento materno exclusivo e total. O tempo total de amamentação foi testado por meio do teste de Wilcoxon considerando localização do domicílio, idade materna, situação conjugal materna, paridade, número de consultas no pré-natal e orientações profissionais sobre amamentação durante o pré-natal. Resultados: $O$ tempo mediano de aleitamento materno exclusivo e total foi de cinco e nove meses, respectivamente. A amamentação foi menos prolongada entre as mães mais novas, primíparas, residentes na zona urbana e que realizaram menos de seis consultas de pré-
} 
natal. As mães que receberam orientações sobre a importância de amamentar até os dois anos de idade ou mais e sobre os prejuízos do uso de chupeta ou bico apresentaram maiores medianas de tempo de aleitamento materno total. Conclusão: As estimativas destacam tempos de aleitamento materno exclusivo e total aquém do esperado, sendo a duração influenciada por características maternas e pela adequação do pré-natal no número de consultas e nas orientações sobre amamentação.

Palavras-chave: Aleitamento Materno. Atenção Primária à Saúde. Estratégia Saúde da Família. Cuidado Pré-Natal. Promoção da Saúde

\section{Abstract}

Objective: To estimate the breastfeeding time among children cared for at the public health network and to check differences according to maternal characteristics and prenatal care (number of consultations and professional orientations), whose data were retrospectively collected in 2011. Methods: Cross-sectional research performed in 16 Primary Family Health Care Units with 204 children born in 2009, which took place in a town in the Brazilian Northeast. The collection was conducted retrospectively in 2011, by applying a questionnaire to the mothers of the children. We used survival analysis to estimate the average length of exclusive and total breastfeeding. Total breastfeeding time was assessed through the Wilcoxon test considering household location, maternal age, maternal marital status, parity, number of prenatal consultations and professional orientations on breastfeeding during prenatal. Results: The average times of exclusive and total breastfeeding were five and nine months, respectively. Breastfeeding was less prolonged among younger and primiparous mothers living in the urban area and that accomplished less than six prenatal consultations. Mothers who received orientations on the importance of breastfeeding up to two years of age or over and about the harms caused by pacifier or nipple use presented higher averages of total breastfeeding time. Conclusion: Estimations highlight exclusive and total breastfeeding times below expectations, where length is influenced by maternal characteristics and prenatal adequacy in the number of consultations and orientations on breastfeeding.

Keywords: Breast Feeding. Primary Health Care. Family Health Strateg. Prenatal Care. Health Promotion 


\section{INTRODUÇÃO}

O aleitamento materno contribui de maneira efetiva no adequado crescimento e desenvolvimento da criança; fortalece o sistema imunológico; previne a desnutrição, doenças infecciosas e alergias; facilita a cognição; reforça o vínculo, afeto e proteção mãe-filho e protege a saúde futura. Inclui benefícios maternos, como menor probabilidade de desenvolver câncer de mama e de ovário, proteção contra o diabetes, favorecendo a perda de peso no pós-parto e propiciando maior rapidez na involução uterina. Não representa custo financeiro para as famílias e constitui a maior e mais econômica intervenção de redução da morbimortalidade infantil, impactando positivamente nos indicadores de saúde de toda a sociedade. ${ }^{1-5}$

A Organização Mundial da Saúde (OMS) recomenda a prática de aleitamento materno exclusivo (AME) durante os primeiros seis meses de vida e a manutenção da amamentação associada aos alimentos complementares até o segundo ano de vida ou mais. Indicadores como AME, aleitamento materno predominante, aleitamento materno complementado e aleitamento materno total (AMT) são recomendados como indicadores de avaliação das práticas alimentares nos primeiros anos de vida. ${ }^{6}$

Os dados mais recentes sobre o aleitamento materno no Brasil são da II Pesquisa de Prevalência de Aleitamento Materno (PPAM) nas capitais brasileiras e Distrito Federal, em 2009, a qual descreveu prevalências de 41,0\% de AME em menores de seis meses de idade e de 58,7\% de aleitamento materno na faixa etária de nove a 12 meses. No Nordeste, essas taxas foram de $37,0 \%$ e $59,1 \%$, respectivamente. ${ }^{7}$

Para que o início e a manutenção do aleitamento materno tenham sucesso, as mulheres necessitam de suporte ativo durante todo o ciclo gravídico puerperal. Todos os profissionais de saúde devem estar comprometidos com a promoção da amamentação, oferecendo informações apropriadas e criando habilidades para sua prática. ${ }^{8}$ A rede básica de saúde destaca-se por ser a principal responsável pela assistência antes e depois do parto, sendo indispensável na promoção, proteção e apoio ao aleitamento materno. ${ }^{9}$ As informações e o suporte relacionados à amamentação oferecido às gestantes no decorrer do pré-natal ajudam a mulher na compreensão de todos os aspectos que envolve tal prática, fortalece a confiança e as capacidades maternas em amamentar e tem efeito positivo nos comportamentos das mulheres que amamentam. Essas intervenções podem ter implicações positivas sobre o início, a exclusividade e a duração do aleitamento materno.9-11

Com vistas à melhoria dos indicadores relacionados à amamentação e buscando explorar o nível primário de apoio a esta prática, municípios do estado do Rio de Janeiro adotaram, desde 
1999, a iniciativa Unidade Básica Amiga da Amamentação. Esta propõe a implantação de "Dez Passos para o Sucesso da Amamentação", trazendo resultados favoráveis para a prática do AME. ${ }^{12}$ Os passos para a unidade de saúde se tornar amiga da amamentação incluem a capacitação dos profissionais e as orientações focadas nessa prática. ${ }^{13}$ Considera-se que o manejo clínico adequado da amamentação por parte dos profissionais da saúde é fator importante e decisivo nas práticas de aleitamento materno. 3,9 Estudos desenvolvidos no Brasil com foco nos fatores relacionados à duração do AMT ainda são poucos, ${ }^{5}$ especialmente considerando as condutas adotadas pelos profissionais da rede básica de saúde durante o prénatal. ${ }^{14} \mathrm{~A}$ compreensão desses fatores pode subsidiar o processo de elaboração de programas e ações de promoção do aleitamento materno. ${ }^{5}$

O presente trabalho teve como objetivo estimar o tempo de duração do aleitamento materno entre crianças nascidas em 2009 atendidas na rede pública de saúde e verificar diferenças segundo características maternas e da atenção pré-natal (número de consultas e orientações profissionais).

\section{MÉTODO}

Estudo transversal analítico desenvolvido no período de julho a agosto de 2011, no município de Queimadas, Paraíba, nas 16 Unidades Básicas de Saúde da Família (UBSF) do município, das quais 11 são rurais e cinco urbanas e representam cobertura de 100\% da população. Essas unidades de saúde são responsáveis por prestar atenção pré-natal às gestantes de baixo risco.

Foram consideradas elegíveis para o estudo todas as crianças nascidas no ano de 2009 e residentes no município de Queimadas, Paraíba, com idade entre 18 e 30 meses de idade na época da coleta dos dados. Crianças de mães com as quais não foi possível realizar contato foram consideradas perdas. Excluíram-se crianças de mães que não realizaram consulta prénatal em UBSF do município, crianças cujas mães engravidaram após o filho nascido em 2009, crianças de mães que possuíam idade inferior a 18 anos no momento da coleta de dados, crianças com malformações congênitas graves, crianças gêmeas e casos de óbito (figura 1).

As informações foram coletadas nas UBSF e/ou no próprio domicílio, por profissionais e estudantes da área de saúde devidamente treinados, supervisionados e com auxílio de um manual de instruções. O estudo piloto foi realizado com população semelhante à do estudo em outro município do mesmo estado. 
O instrumento de coleta de dados constou de um questionário pré-codificado, com questões fechadas, contendo informações relacionadas à localização do domicílio (rural, urbana), idade materna ( $\geq 20$ anos, 18 a 19 anos), situação conjugal materna (com companheiro, sem companheiro), paridade (1 filho, 2 ou mais filhos) e número de consultas no pré-natal ( $\geq 6$, <6). As mães também foram questionadas sobre o recebimento de orientações de promoção da amamentação pelos profissionais de saúde durante as consultas de pré-natal, a saber: i) importância de iniciar a amamentação na primeira hora de vida; ii) como resolver dificuldades com relação à amamentação; iii) posições adequadas da criança e da mãe para o aleitamento materno; iv) como retirar o leite materno; v) importância da amamentação até os dois anos de idade ou mais; vi) importância de amamentar sempre que a criança quiser; vii) prejuízos do uso de mamadeira; e viii) prejuízos do uso de chupeta ou bico.

Figura 1. Fluxograma de seleção da população de estudo, para descrever o aleitamento materno entre crianças de 18-30 meses de idade. Queimadas, Paraíba, 2011 ( $N=204)$.

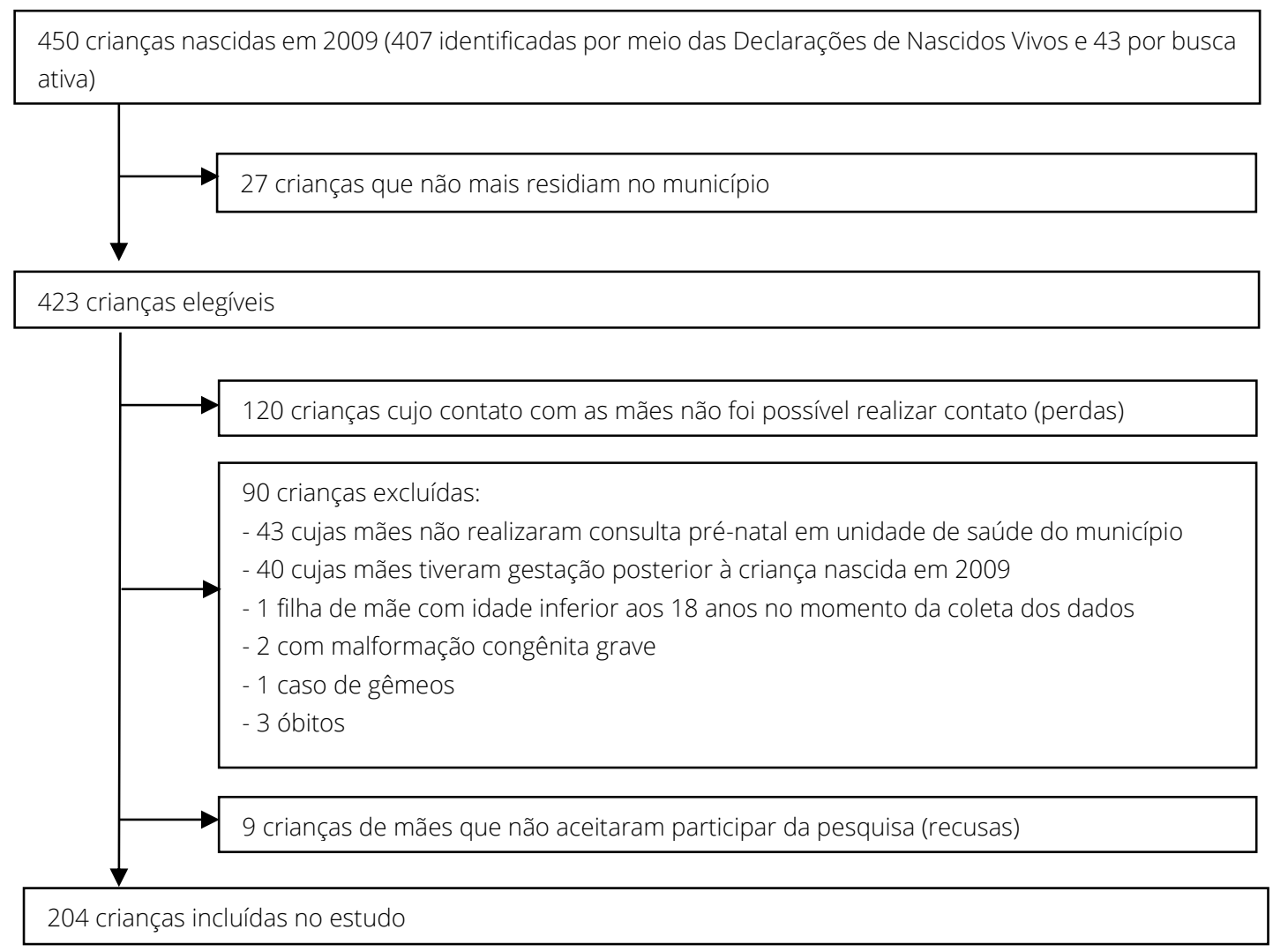


Foram utilizadas as definições de aleitamento materno adotadas pela OMS. ${ }^{6}$ Para definir a duração do AME considerou-se o período no qual a criança permaneceu recebendo somente leite materno, direto da mama ou ordenhado, ou leite humano de outra fonte, sem outros líquidos ou sólidos, com exceção de gotas ou xaropes contendo vitaminas, sais de reidratação oral, suplementos minerais ou medicamentos. O tempo de AMT foi calculado considerando-se o período em que a criança recebeu leite materno (direto da mama ou ordenhado), independentemente de receber ou não outros alimentos. ${ }^{6}$

Os dados foram digitados no programa Excel 2010, com dupla digitação dos questionários, para comparação e detecção de possíveis erros. Foi utilizado o aplicativo Validate do programa Epi-Info para verificar a consistência dos dados.

Utilizou-se a análise de sobrevida através do método atuarial para estimar a duração mediana das práticas do aleitamento materno, considerando pelo menos um dia de amamentação para todas as crianças do estudo. Para a apresentação gráfica, foram utilizados os diferenciais das medianas mensais das práticas de aleitamento materno resultantes da análise de sobrevida.

O teste de Shapiro Wilk foi utilizado para testar a suposição de normalidade das variáveis. Utilizou-se o teste de Wilcoxon para analisar diferenças na duração do aleitamento materno total segundo variáveis explanatórias estudadas (localização do domicílio, idade materna, situação conjugal materna, paridade, número de consultas no pré-natal, condutas profissionais de promoção do aleitamento materno durante o pré-natal). As análises estatísticas foram realizadas no programa estatístico R v2.10.0, adotando-se o nível de significância de 5\%.

O projeto de pesquisa foi aprovado pelo Comitê de Ética em Pesquisa da Universidade Estadual da Paraíba, Aprovação CAAE 0170.0.133.000-11.

\section{RESULTADOS}

Comparadas as curvas de sobrevida das diferentes práticas do aleitamento materno nas 204 crianças do estudo (figura 2), verificou-se declínio dos segmentos iniciais, com alta taxa de desmame desde os primeiros meses de vida e redução da oferta do aleitamento exclusivo. 0 tempo mediano do AME e do AMT foi de cinco e nove meses, respectivamente. Ao completarem seis meses de vida, 56,9\% das crianças ainda mamavam, sendo que, no mesmo período, o percentual de crianças em AME foi de 10,0\%. Aos 24 meses, 20,0\% das crianças ainda estavam sendo amamentadas. 
Figura 2. Tempo de aleitamento materno (total e exclusivo) e proporção entre crianças de 18-30 meses de idade. Queimadas, Paraíba, 2011 (N=204).

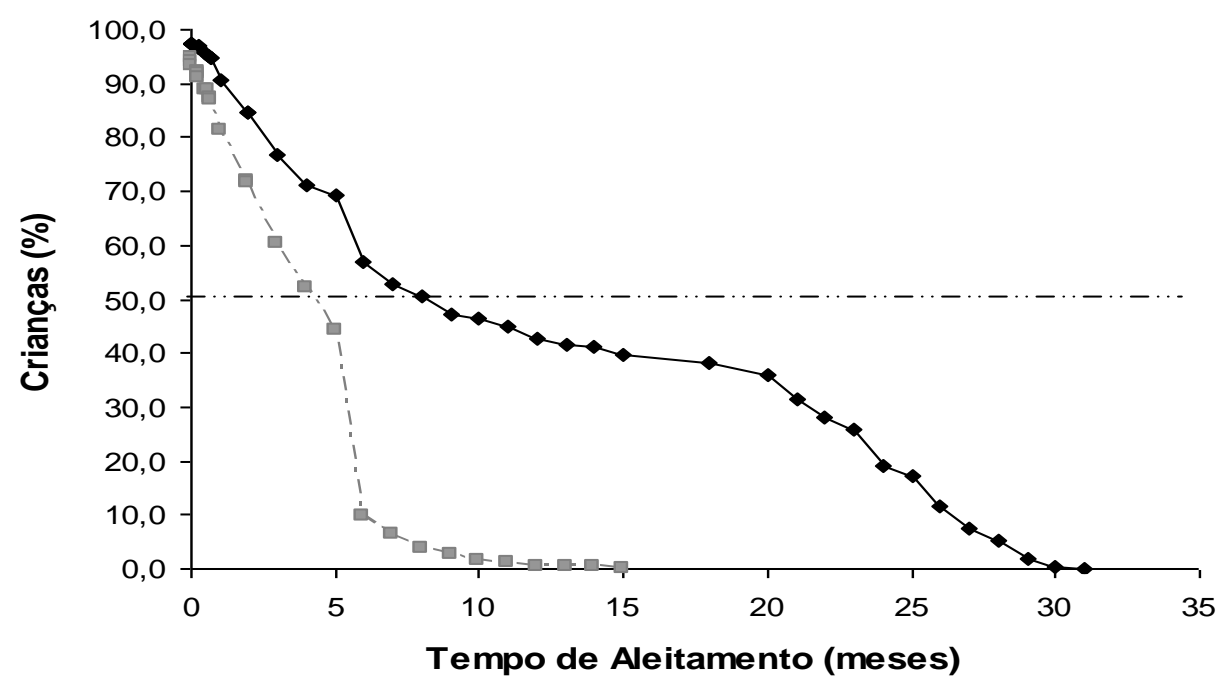

$\longrightarrow$ Aleitamento Total - - - - Aleitamento Exclusivo

A tabela 1 apresenta as diferenças nas medianas dos tempos de AMT, segundo variáveis independentes. Na amostra, 61,8\% dos domicílios localizavam-se na zona rural, 94,1\% das mães eram adultas, 80,9\% viviam com companheiro, 56,9\% deram à luz a dois ou mais filhos e 69,1\% realizaram seis ou mais consultas no pré-natal. A duração do AMT foi maior entre crianças residentes na zona rural, cujas mães tinham idade de 20 anos ou mais, tinham mais de um filho e cujas mães realizaram seis ou mais consultas de pré-natal, quando comparadas às da zona urbana, de mães com menos de 20 anos, filhos únicos e que durante a gravidez realizaram menos de seis consultas pré-natais, respectivamente.

Tabela 1. Tempo de aleitamento materno total entre crianças de 18-30 meses de idade segundo características sociodemográficas maternas. Queimadas, Paraíba, 2011 (N=204).

\begin{tabular}{|c|c|c|c|c|}
\hline Variáveis & $n$ & $\%$ & Mediana de AMT (meses) & p-valor \\
\hline Localização do domicílio & & & & 0,010 \\
\hline Urbana & 78 & 38,2 & 8,0 & \\
\hline Rural & 126 & 61,8 & 9,5 & \\
\hline Idade materna (anos) & & & & 0,001 \\
\hline$\geq 20$ & 192 & 94,1 & 8,5 & \\
\hline 18 a 19 & 12 & 5,9 & 6,0 & \\
\hline Situação conjugal materna & & & & 0,584 \\
\hline Com companheiro & 165 & 80,9 & 9,0 & \\
\hline Sem companheiro & 39 & 18,1 & 7,5 & \\
\hline
\end{tabular}


Tabela 1. Tempo de aleitamento materno total entre crianças de 18-30 meses de idade segundo características sociodemográficas maternas. Queimadas, Paraíba, 2011 (N=204). (cont.)

\begin{tabular}{lcccc}
\hline Variáveis & $\mathrm{n}$ & $\%$ & Mediana de AMT (meses) & p-valor \\
\hline Paridade & & & & $<0,001$ \\
$\quad 1$ filho & 88 & 43,1 & 6,0 & \\
2 ou mais filhos & 116 & 56,9 & 14,5 & 0,030 \\
Número de consultas no pré-natal & & & & \\
$\geq 6$ & 141 & 69,1 & 11,0 & \\
$<6$ & 63 & 30,9 & 7,0 & \\
\hline
\end{tabular}

AMT - aleitamento materno total.

Segundo o relato materno, as orientações menos praticadas pelos profissionais de saúde foram sobre como resolver dificuldades com relação à amamentação (34,3\%) e como retirar o leite materno (37,3\%). Mães que referiram ter recebido orientações sobre a importância de amamentar até os dois anos de idade ou mais $(p=0,004)$ e sobre os prejuízos do uso de chupeta ou bico $(p=0,041)$ apresentaram maiores medianas de tempo de AMT do que as que relataram não ter recebido tais orientações (tabela 2).

Tabela 2. Tempo de aleitamento materno total entre crianças de 18-30 meses de idade segundo orientações sobre amamentação realizadas pelos profissionais durante o pré-natal. Queimadas, Paraíba, 2011 (N=204).

\begin{tabular}{|c|c|c|c|c|}
\hline Orientações & $\mathrm{n}$ & $\%$ & Mediana de AMT (meses) & p-valor \\
\hline Sobre a importância de iniciar a amamentação & & & & 0,680 \\
\hline \multicolumn{5}{|l|}{ ao peito na primeira hora de vida } \\
\hline Sim & 175 & 85,8 & 8,5 & \\
\hline Não & 29 & 14,2 & 9,0 & \\
\hline Sobre como resolver dificuldades com relação & & & & 0,216 \\
\hline \multicolumn{5}{|l|}{ à amamentação } \\
\hline Sim & 134 & 65,7 & 9,0 & \\
\hline Não & 70 & 34,3 & 8,0 & \\
\hline \multicolumn{4}{|l|}{ mãe para o aleitamento materno } & 0,344 \\
\hline $\operatorname{sim}$ & 145 & 71,1 & 8,0 & \\
\hline Não & 59 & 28,9 & 9,0 & \\
\hline Sobre como retirar o leite materno & & & & 0,097 \\
\hline Sim & 128 & 62,7 & 8,0 & \\
\hline Não & 76 & 37,3 & 9,0 & \\
\hline Sobre a importância do aleitamento materno & & & & 0,004 \\
\hline \multirow{3}{*}{\multicolumn{5}{|c|}{$\begin{array}{l}\text { exclusivo durante os seis primeiros meses de } \\
\text { vida e a manutenção da amamentação até os } \\
\text { dois anos de idade ou mais }\end{array}$}} \\
\hline & & & & \\
\hline & & & & \\
\hline Sim & 139 & 68,1 & 10,0 & \\
\hline Não & 65 & 31,9 & 6,0 & \\
\hline
\end{tabular}


Tabela 2. Tempo de aleitamento materno total entre crianças de 18-30 meses de idade segundo orientações sobre amamentação realizadas pelos profissionais durante o pré-natal. Queimadas, Paraíba, 2011 ( $N=204)$. (cont.)

\begin{tabular}{lcccc}
\hline Orientações (cont.) & $\mathrm{n}$ & $\%$ & Mediana de AMT (meses) & $\mathrm{p}$-valor \\
\hline Sobre a importância de amamentar sempre & & & & 0,185 \\
que a criança quiser & 139 & 68,1 & 8,0 & \\
$\quad$ Sim & 65 & 31,9 & 9,0 & 0,126 \\
$\quad$ Não & & & & \\
Sobre os prejuízos do uso de mamadeira & 150 & 73,5 & 6,0 & 0,041 \\
$\quad$ Sim & 54 & 26,5 & & \\
Não & 149 & 73,0 & 9,0 & \\
Sobre os prejuízos do uso de chupeta ou bico & 55 & 27,0 & 6,0 & \\
$\quad$ Sim & & &
\end{tabular}

AMT - aleitamento materno total.

\section{DISCUSSÃO}

O tempo mediano de AME no presente estudo (5 meses) foi superior ao encontrado na Pesquisa Nacional de Demografia e Saúde da Criança e da Mulher (PNDS) de 2006 (2,2 meses) $)^{15}$ e na PPAM de 2009, tanto em relação ao dado nacional (1,8 meses) quanto ao da Região Nordeste $\left(1,16\right.$ meses). ${ }^{7}$ Diante do contexto evidenciado nessas pesquisas ${ }^{7,15}$ e em outros estudos, ${ }^{16-18}$ considera-se que o tempo de AME no município de Queimadas foi um resultado favorável.

A mediana de AMT foi de nove meses, mostrando-se semelhante à evidenciada no âmbito nacional em $2006\left(9,1\right.$ meses) ${ }^{15}$ e inferior à que foi verificada em 2009 para o conjunto das capitais brasileiras e Distrito Federal (11,2 meses) e para a Região Nordeste (11,4 meses). ${ }^{7}$ Considerando a capacidade de contribuir com a promoção do aleitamento materno, ressalta-se a necessidade de as mães serem continuamente apoiadas após a alta hospitalar na Estratégia Saúde da Família (ESF), juntamente com a atuação efetiva dos ACS.9,16,19 Estudos no Brasi|3,18,20,21 têm mostrado medianas de AMT divergentes, com valores superiores a 365 dias em algumas localidades ${ }^{3,20}$ e inferiores em outras. ${ }^{18,21}$ A diversidade observada no tempo de AMT sugere a importância do incentivo à realização de diagnósticos situacionais locais, no sentido de subsidiar o planejamento de intervenções apropriadas.

Alguns fatores apresentaram diferenças no tempo de AMT das crianças estudadas, como a idade materna de 18 a 19 anos e a primiparidade. Esses resultados são similares aos de estudos anteriores, que mostraram maior probabilidade de interrupção do aleitamento materno em mães mais jovens ${ }^{10,17,21,22}$ e entre aquelas que estavam parindo o primeiro filho. 4,23 
De forma semelhante, a influência da idade materna e da paridade na duração do AME foi constatada em revisão sistemática da literatura. ${ }^{24} \mathrm{O}$ maior tempo de amamentação entre mulheres de maior idade pode estar relacionado ao conhecimento e experiência em relação ao aleitamento materno. ${ }^{10} \mathrm{~A}$ menor frequência de amamentação nas mães primíparas pode estar relacionada à associação da experiência prévia com a amamentação com a maior duração dessa prática e do $\mathrm{AME}^{4,13}$ o que reforça a importância de apoiar as mulheres que ainda não amamentaram ou que o fizeram por pouco tempo, no caso dos filhos anteriores. ${ }^{13}$

Apesar da redução das diferenças na prevalência de amamentação entre áreas urbanas e rurais no Brasil, ${ }^{25}$ os dados do presente estudo mostraram, à semelhança de outro, ${ }^{20}$ que mães residentes em áreas rurais apresentam maior mediana de aleitamento materno total, comparadas com as da área urbana. É possível que nas zonas rurais haja manutenção de padrões culturais que preservam rotinas de trabalho fora do lar mais flexíveis, e persistência de estruturas tradicionais de apoio à família e pressões da modernização menos vigorosas, fatores que podem facilitar a manutenção do aleitamento materno. ${ }^{25}$

A assistência pré-natal mostrou-se de grande importância para o sucesso da amamentação, com duração mais elevada entre as mães que realizaram seis ou mais consultas, o que corrobora achados prévios também no Nordeste do Brasil. ${ }^{5,18} \mathrm{Em}$ um estudo canadense de abrangência nacional, constatou-se que o cuidado pré-natal provido por médico da família ou parteira impacta positivamente no comportamento da mãe em relação à amamentação. ${ }^{11} \mathrm{O}$ menor tempo de amamentação entre mulheres com menos consultas de pré-natal pode estar relacionado ao menor acesso às informações sobre aleitamento. ${ }^{26}$

Apesar de o sucesso do aleitamento materno não estar meramente relacionado ao acesso à informação, uma vez que as práticas de amamentação são afetadas por ampla gama de fatores históricos, socioeconômicos, culturais e individuais que incluem características maternas (idade, trabalho, escolaridade, paridade, tipo de parto), renda familiar e zona de residência, ${ }^{2,26} \mathrm{O}$ presente estudo confirmou que a adoção de orientações relacionadas à amamentação (sobre a importância de amamentar até os dois anos de idade ou mais e dos prejuízos do uso de chupeta ou bico) pode contribuir positivamente no tempo de aleitamento materno. Esses resultados convergem com os de outros estudos nacionais e internacionais, que identificaram associação positiva da amamentação com a adoção de práticas para sua promoção na rede básica de saúde, , ,9,13,27-29 especialmente quando realizadas por profissionais capacitados, 8,9,30,31 reforçando os pressupostos que atestam a importância de investimentos nas mesmas. ${ }^{26} \mathrm{~A}$ pouca quantidade de estudos sobre o reflexo de tais orientações desenvolvidas na atenção básica ${ }^{27}$ torna relevantes os resultados encontrados. 
O fato de ter sido a orientação sobre a importância do AME durante os seis primeiros meses de vida e a manutenção da amamentação até os dois anos de idade ou mais um dos fatores que marcou diferenças no tempo de aleitamento materno é consistente com os achados de uma revisão da literatura, na qual o incentivo do AME pelos profissionais de saúde foi destacado dentre os fatores mais relevantes na prática do aleitamento materno, influenciando os conhecimentos, a confiança e a segurança das mulheres na hora de amamentar. Ainda, os autores apontaram o uso de chupeta como importante responsável pelo desmame precoce, ${ }^{32}$ estando igualmente em sintonia com a influência desse hábito na duração do AMT reportada no atual estudo. Assim, torna-se essencial informar às mães sobre os prejuízos do uso de chupeta na frequência das mamadas e, portanto, na produção de leite. ${ }^{24,32}$ A percepção materna da importância dos profissionais de saúde no incentivo e na promoção do aleitamento materno agrega maior valor à participação ativa dos mesmos como forma de empoderar a mulher e aderir a práticas adequadas de amamentação. ${ }^{33}$

A leitura dos resultados do atual estudo deve considerar os vieses próprios dos estudos transversais, em especial pela impossibilidade de estabelecer causalidade e por tratar o aleitamento materno com a utilização de informações recordatórias que podem levar a erros de memória do entrevistado. Contudo, a faixa etária estudada na presente investigação, de crianças da última gestação que tinham no máximo dois anos e meio, pode minimizar o viés de recordatório materno. Além disso, o treinamento dos entrevistadores e o uso de um manual de instruções envolveram habilidades de comunicação e empatia fundamentais para o controle de possíveis vieses de memória associados às questões retrospectivas. Por outro lado, os dados sobre a duração da amamentação foram analisados através da técnica da análise de sobrevida, a qual possibilita uma estimativa longitudinal com dados coletados transversalmente.

Adicionalmente, as limitações do presente estudo devem considerar que sua realização durante um ano e com gestantes de um município impossibilita a extrapolação dos resultados para a realidade brasileira. E ainda, as perdas, embora referidas à população elegível/total, redundam em restrições relacionadas à validade dos resultados. Deve-se considerar que, para avaliar o efeito das orientações feitas pelos profissionais de saúde, seria necessário utilizar um grupo controle. Apesar das limitações, os achados apresentam contribuição relevante, ao mostrarem os benefícios, no aleitamento materno, de práticas simples de apoio à amamentação durante o pré-natal.

\section{CONCLUSÃO}


A duração do aleitamento materno exclusivo e total está aquém do esperado, sendo a deste último influenciada por características maternas (zona de residência, idade materna, número de filhos), pela adequação do número de consultas de pré-natal e pelo recebimento de orientações sobre amamentação. Para a ampliação dessas práticas, deve-se priorizar a capacitação dos profissionais de saúde sobre a importância do aleitamento e o desenvolvimento de iniciativas que apoiem a amamentação durante o pré-natal, como a Unidade Básica Amiga da Amamentação.

\section{REFERÊNCIAS}

1. Victora CG, Barros AJD, França GVA, Bahl R, Rollins NC, Horton S, et al. Breastfeeding in the 21st century: epidemiology, mechanisms, and lifelong effect. Lancet 2016; 387(10017):475-90. http://dx.doi.org/10.1016/S0140-6736(15)01024-7.

2. Caminha MFC, Serva VB, Arruda IKG, Batista FM. Aspectos históricos, científicos, socioeconômicos e institucionais do aleitamento materno. Rev Bras Saúde Matern Infant. 2010; 10(1):25-37. http://dx.doi.org/10.1590/S1519-38292010000100003.

3. Tamasia GA, Venâncio SI, Saldiva SRDM. Situation of breastfeeding and complementary feeding in a medium-sized municipality in the Ribeira Valley, São Paulo. Rev Nutr. 2015; 28(2):14353. http://dx.doi.org/10.1590/1415-52732015000200003.

4. Wenzel D, Souza SB. Fatores associados ao aleitamento materno nas diferentes Regiões do Brasil. Rev Bras Saúde Matern Infant. 2014; 14(3):241-9. http://dx.doi.org/10.1590/S151938292014000300005.

5. Mendes SC, Lobo IKV, Sousa SQ, Vianna RPT. Fatores relacionados com uma menor duração total do aleitamento materno. Cienc Saude Colet. 2017; 1:16457. http://dx.doi.org/10.1590/141381232018245.13772017.

6. World Health Organization. Indicators for assessing infant and young child feeding practices: conclusions of a consensus. Washington DC: World Health Organization; 2008.

7. Brasil. Ministério da Saúde. II Pesquisa de Prevalência de Aleitamento Materno nas Capitais Brasileiras e Distrito Federal. Brasília: Ministério da Saúde; 2009.

8. Cruz SH, Germano JÁ, Tomasi E, Facchini LA, Piccini RX, Thumé E. Orientações sobre amamentação: a vantagem do Programa de Saúde da Família em municípios gaúchos com mais de 100.000 habitantes no âmbito do PROESF. Rev Bras Epidemiol. 2010; 13(2):259-67. http://dx.doi.org/10.1590/S1415790X2010000200008.

9. Vasquez J, Dumith SC, Susin LRO. Aleitamento materno: estudo comparativo sobre o conhecimento e o manejo dos profissionais da Estratégia Saúde da Família e do Modelo Tradicional. Rev Bras Saúde Matern Infant. 2015; 15(2):181-92. http://dx.doi.org/10.1590/S1519-38292015000200004.

10. Margotti E, Epifanio M. Aleitamento materno exclusivo e a Escala de Autoeficácia na Amamentação. Rev Rene 2014; 15(5):771-9. http://dx.doi.org/10.15253/2175-6783.2014000500006. 
11. Costanian C, Macpherson AK, Tamim H. BMC Inadequate prenatal care use and breastfeeding practices in Canada: a national survey of women. BMC Pregnancy and Childbirth 2016; 16:100. http://dx.doi.org/10.1186/s12884-016-0889-9.

12. Alves ALN, Oliveira MIC, Moraes JR. Iniciativa Unidade Básica Amiga da Amamentação e sua relação com o aleitamento materno exclusivo. Rev Saúde Pública 2013; 47(6):1130-40. http://dx.doi.org/10.1590/S0034-8910.2013047004841.

13. Pereira RSV, Oliveira MIC, Andrade CLT, Brito AS. Fatores associados ao aleitamento materno exclusivo: o papel do cuidado na atenção básica. Cad Saúde Pública 2010; 26(12):2343-54. http://dx.doi.org/10.1590/S0102-311X2010001200013.

14. Uema RTB, Souza SNDH, Mello DF, Capellini VK. Prevalência e fatores associados ao aleitamento materno no Brasil entre os anos 1998 e 2013: revisão sistemática. Semina: Ciências Biológicas e da Saúde 2015; 36(1 Supl):349-62. http://dx.doi.org/10.5433/1679-0367.2014v35n2p349.

15. Brasil. Ministério da Saúde. Pesquisa Nacional de Demografia e Saúde da Criança e da Mulher - PNDS 2006: dimensões do processo reprodutivo e da saúde da criança. Brasília: Ministério da Saúde; 2009.

16. Brecailo MK, Corso ACT, Almeida CCB, Schmitz BAS. Fatores associados ao aleitamento materno exclusivo em Guarapuava, Paraná. Rev Nutr. 2010; 23(4):553-63. http://dx.doi.org/10.1590/S141552732010000400006.

17. Warkentin S, Taddei JAAC, Viana KJ, Colugnati FAB. Exclusive breastfeeding duration and determinants among Brazilian children under two years of age. Rev Nutr. 2013; 26(3):259-69. http://dx.doi.org/10.1590/S1415-52732013000300001.

18. Oliveira MGOA, Lira PIC, Filho MB, Lima MC. Fatores associados ao aleitamento materno em dois municípios com baixo índice de desenvolvimento humano no Nordeste do Brasil. Rev Bras Epidemiol. 2013; 16(1):178-89. http://dx.doi.org/10.1590/S1415-790X2013000100017.

19. Coutinho SB, Lira PI, Lima MC, Frias PG, Eickmann SH, Ashworth A. Promotion of exclusive breastfeeding at scale within routine health services: impact of breast-feeding counselling training for community health workers in Recife, Brazil. Public Health Nut. 2014; 17(4):948-55. http://dx.doi.org/10.1017/S1368980013001833.

20. Demétrio F, Pinto EJ, Assis AMO. Fatores associados à interrupção precoce do aleitamento materno: um estudo de coorte de nascimento em dois municípios do Recôncavo da Bahia, Brasil. Cad Saúde Pública 2012; 28(4):641-50. http://dx.doi.org/10.1590/S0102-311X2012000400004.

21. Caminha MFC, Filho MB, Serva VB, Arruda IKG, Figueiroa JN, Lira PIC. Tendências temporais e fatores associados à duração do aleitamento materno em Pernambuco. Rev Saúde Pública 2010; 44(2):2408. http://dx.doi.org/10.1590/S0034-89102010000200003.

22. Neves ACM, Moura EC, Santos W, Carvalho KMV. Factors associated with exclusive breastfeeding in the Legal Amazon and Northeast regions, Brazil, 2010. Rev Nutr. 2014; 27(1):81-95. http://dx.doi.org/10.1590/1415-52732014000100008.

23. Martins CC, Vieira GO, Vieira TO, Mendes CM. Fatores de riscos maternos e de assistência ao parto para interrupção precoce do aleitamento materno exclusivo: estudo de coorte. Rev Baiana Saude Publica 2011; 35(Supl 1):167-8. 
24. Rocha IS, Lolli LF, Fujimaki M, Gasparetto A, Rocha NB. Influência da autoconfiança materna sobre o aleitamento materno exclusivo aos seis meses de idade: uma revisão sistemática. Cienc Saude Colet. 2018; 23(11):3609-19. http://dx.doi.org/10.1590/1413-812320182311.20132016.

25. Wenzel D, Souza SB. Prevalência do aleitamento materno no Brasil segundo condições socioeconômicas e demográficas. Rev Bras Crescimento Desenvolv Hum. 2011; 21(2):251-8. https://doi.org/10.7322/jhgd.20013.

26. Rollins NC, Lutter CK, Bhandari N, Hajeebhoy N, Horton S, Martines JC, et al. Why invest and what it will take to improve breastfeeding practices? Lancet 2016; 387(10017):491-504. http://dx.doi.org/10.1016/S0140-6736(15)01044-2.

27. Alves JS, Oliveira MIC, Rito RWF. Orientações sobre amamentação na atenção básica de saúde e associação com o aleitamento materno exclusivo. Cienc Saude Colet. 2018; 23(4):1077-88. http://dx.doi.org/10.1590/1413-81232018234.10752016.

28. Busch D, Nassar L, Silbert-Flagg J. The Necessity of Breastfeeding - Promoting Breastfeeding in the Primary Care Setting; A Community Pilot Project Applying the Tri-Core Breastfeeding Model: Beyond the Basics. J Preg Child Health 2015; 2(3):1000158. http://dx.doi.org/10.4172/2376-127X.1000158.

29. Wen LM, Simpson JM, Rissel C, Baur LA. Awareness of Breastfeeding Recommendations and Duration of Breastfeeding: Findings from the Healthy Beginnings Trial. Breastfeeding Med. 2012; 7(4):223-9. http://dx.doi.org/10.1089/bfm.2011.0052.

30. Almeida JM, Luz SAB, Ued FV. Apoio ao aleitamento materno pelos profissionais de saúde: revisão integrativa da literatura. Rev Paul Pediatr. 2015; 33(3):355-62. http://dx.doi.org/10.1016/j.rpped.2014.10.002.

31. Almeida ISA, Pugliesi Y, Rosado LEP. Estratégias de promoção e manutenção do aleitamento materno baseadas em evidência: revisão sistemática. Femina 2015; 43(3):97-103.

32. Lima APC, Nascimento DS, Martins MMF. A prática do aleitamento materno e os fatores que levam ao desmame precoce: uma revisão integrativa. J Health Biol Sci. 2018; 6(2):189-96. http://dx.doi.org/10.12662/2317-3076jhbs.v6i2.1633.p189-196.2018.

33. Pontes AM, Lucena KDT, Silva ATMC, Almeida LR, Deininger LSC. As repercussões do aleitamento materno exclusivo em crianças com baixo peso ao nascer. Saúde Debate 2013; 37(97):354-61.

Conflito de interesses: Os autores declaram não haver conflitos de interesses.

Recebido: 05 de junho de 2019

Revisado: 17 de julho de 2019

Aceito: 28 de agosto de 2019 